\title{
Beitrag zur Beurtheilung des Werthes der verschiedenen Quecksilberpräparate in der Syphilistherapie.
}

Von

Dr. Erich Lexer in Würzburg.

Es hat sich im Laufe der Jahrhunderte auf dem Gebiete der Syphilistherapie die Inunctionseur mit Quecksilbersalben von ursprünglich sehr zweifelhaften und grausamen Methoden, insbesondere auch durch das Verdienst von Sigmund in diesem Jahrhundert zu der besten und sichersten Heilmethode der Syphilis ontwickelt. Aber seit ihrer Existenz hatte die Inunctionscur ihre gewaltigen Gegner. Abgesehen von früherer Zeit, wo das Suchen nach nenen Mitteln nur zu gerechtfertigt war dureh die Angst und Scheu von Laien und Aerzten vor den oft mit entsetzlichen Folgen begleiteten, unvernünftig gehandhabten Inunctionseuren, findet man namentlich in neuerer Zeit einen beständigen Kampf gegen diese Therapie, ein beständiges Suchen und Versuchen, durch andere Mittel und Methoden die veraltete Methode der Syphilisbehandlung durch Einreibungen mit grauer Salbe zu stürzen und Besseres an ihre Stelle zu setzen.

So geht schon seit mehreren Jahrzehnten das Streben der Forscher und Aerzte dabin, eine chemische Verbindung des Quecksilbers zn finden, die neben starker Entfaltnng ihrer Wirkung auf das syphilitische Gift vom Organismus gut vertragen wird und durch die Art der Anwendung jene Nachtheile entbehrt, die bei den Einreibungen theils nicht vermieden, theils durch Unachtsamkeit herrorgerufen werden können. 
Von diesen Schattenseiten der Inunctionen steht der von alter Zeit her schon beobachtete Speichelfluss an der Spitze, den man sich damals sogar bemühte hervorzurufen, da man in ihm die Abflussquelle des Syphilisgiftes sah, der aber heutzutage durch vorsichtige Behandlung auf ein Minimum beschränkt wird. Man behauptet ferner (Rindfleisch, Arch. f. Derm. u. Syph. 1870; Fleischer, Röhrig), das Quecksilber köune durch die unversehrte Haut nicht eindringen; wenn dasselbe also bei Einreibungen in den Organismus gelangt, so könne dies nur in Dampfform und durch die Athmungsorgane geschehen, zumal ja bekannt ist, dass das Quecksilber schon bei gewöhnlicher Temperatur verdampft, um so mehr also beim Erwärmen während der Einreibungen. Dabei setzt es sich am Zahnfleisch nịeder und ruft durch Reizung Affectionen der Mundschleimbant hervor. Nach Cohnheim kann es nicht mit Sicherheit bestimmt werden, wie grosse Quantitäten Quecksilber bei den Einreibungen in den Organismus gelangen; möglich ist es, dass das Quecksilber in Dampform die Hant durchdringt, wie dies ja für viele Dämpfe und Gase nachgewiesen ist. Lesser bemerkt in seinem Lehrbuche der Hant- und Geschlechtskrankheiten zu dieser Frage: "Dass eine Resorption wirklich stattfindet, darüber kann ein Zweifel nicht bestehen, denn manchmal gelingt es schon 24 Stunden nach der ersten Einreibung, Quecksilber im Urin nachzuweisen; wahrscheinlich geschieht diese Resorption im Wesentlichen in den Ausführungsgängen der Schweiss- und Talgdrüsen", wie dies auch durch anatomische Untersuchung excidirter Hautstückchen nachgewiesen wurde, nenerdings von Pinner (Therap. Monatsh. 1889, 7), und spielt die Aufnahme durch die Respiration nur eine ganz untergeordnete Rolle. Weitere Einwürfe gegen die Inunctionen sind folgende: Man kann die richtige Anwendung nicht immer genau controliren, anf empfindlicher reizbarer Haut treten Erytheme und Eczeme auf, die Anwendung selbst ist mühsam, verschwendet Zeit und verunreinigt $W$ äsche und Betten; nicht luetische Personen, welche den nämlichen Krankensaal mit den luetischen bewohnen, können ebenfalls durch Einathmung der Quecksilberdämpfe an Stomatitis erkranken.

Es ist nicht zu verwundern, dass nach diesen Vorwürfen, welche der alten Syphilistherapie gemacht wurden und die ja in 
gewisser Beziehung ihre Berechtigung haben, ein grosser Theil der medicinischen Welt sich den von den Gegnern der Inunctionen aufgestellten und als vorzüglich gepriesenen subcutanen Injectionen mit Qnecksilberpräparaten einerseits, andererseits der innerlichen Verabreichnng von solchen in Pillenform zuwandte. Die Vortheile dieser neuen Methoden liegen auf der Hand, aber es zeigten sich bald auch hier Nachtheile, die denen der Inunctionen nicht gerade um Vieles nachstehen. Vor Allem gewähren sie ja dem Arzte praktische Vortheile; er kann genaue Controle üben betreffs der Qnecksilbermenge, die der Körper des Kranken aufnehmen soll, ferner ist eine ambulante und dabei doch genaue Behandlung möglich, für den Patienten selbst sind alle diese Curen bequemer und angenehmer, und vor Allem hat das Quecksilber weniger Gelegenheit, in die Umgebung zu verdampfen und so bei den Patienten selbst, wie bei anderen die lästigen. Schleimhautaffectionen der Mundhöhle hervorzurufen.

Viele und meist die bedeutendsten dieser neuen Mittel und Methoden wurden bald nach ihrem Entstehen auch auf der hiesigen syphilitischen Klinik versucht, und zwar kamen seit dem 1. Januar 1880

Hydrargyrum peptonatum,

Hydrargyrum formamidatum,

Hydrargyrum oxydulatum tannicum,

Quecksilberchloridchlornatrium,

Hydrargyrum oxydatum carbolicum,

Hydrargyrum salicylicum,

Oleum cinè̃eum

zur Anwendung.

Ueber die Erfahrungen, die mit diesen Präparaten gemacht worden sind, ist theilweise wenigstens in einigen Dissertationen, auf die ich später zurückkomîmen werde, Bericht erstattet. Diese Arbeit, die auf Anregung von Herrn Privatdocenten Dr. Seifert entstand, soll sich mit statistischen Untersuchungen über das Verhältniss der Recidive der Syphilis zu den einzelnen, eben genannten Präparaten beschäftigen, und zwar erstrecken sich diese Studien auf das Material der hiesigen Syphilido-Klinik aus den Jahren vom 1. Januar 1880 bis 1. Januar 1889.

Voraus zu bemerken ist, dass neben der Behandlung mit 
diesen Mitteln immer, gleichsam zur Controle, bei einer Anzahl von Fällen auch Inunctionscuren angewendet wurden, theils gleichzoitig mit anderen Mathoden, theils in kleineren Zwischenräumen, bis ein neues Mittel versucht wurde.

Die Methode, die bei der Einreibungscur in Anwendung kam, ist im Allgemeinen noch die von Sigmund in der Mitte dieses Jahrhunderts angegebene, die ja damals bahnbrechend für die Syphilistherapie wirkte und den Grund zur hentigen Therapie mit grauer Salbe legte. Ehe die specifische Behandlung eingeleitet wird, müssen andere, gleichzeitig bestehende Krankheiten zur Heilung gelangen, selbst leichtere Affectionen müssen beachtet werden, da ja Katarrhe leicht bei der Cur Stomatitis und Salivation, Magen- und Darmaffectionen heftige Diarhöen veranlassen können. Eine gewisse Regelung der Lebensweise, die Sigmund vor Beginn der Cur verlangt, ist wohl in einem Spitale selbstverständlich, ebenso was seine Forderung bezüglich der Ventilation der Räume, in denen sich die Kranken aufhalten, der Zimmertemperatur, der Reinlichkeit und Nahrung der Patienten betrifft. Was die Mundhygiene angeht, so sind die Kranken sowohl zu mehrmals täglich zu erfolgender, gründlicher Reinigung der Zähne angehalten, als zu öfterem Gebrauche von Gurgelwasser und Zahntinctur, namentlich nach dem Essen. Gegen Stomatitis wurde im letzten Jahre Pinseln mit Bromwasserstoffsäure täglich einmal, bei schwereren Fällen zweimal angewandt, unter welcher Behandlung, ohne dass die Cur ausgesetzt zu werden brauchte; baldige Besserung und Heilung eintrat.

Die Menge der täglich angewendeten 33procentigen Quecksilbersalbe beträgt 4.0 , und zwar werden die Einreibungen unter Aufsicht des Wartepersonals in der Weise ausgeführt, dass bei den Weibern am ersten Tage die beiden Unterschenkel, am zweiten die beiden Oberschenkel, am dritten die beiden Arme, am vierten Banch und Brust, am fünften der Rücken eingerieben werden; bei den Männern ist nur der Unterschied, dass Bauch und Brust gesondert eingerieben werden. Jedesmal nach Beendigung eines solchen Cyklus von Einreibungen bekommen die $\mathrm{Pa}$ tienten ein Vollbad. Kinder erhalten je nach dem Alter täglich 0.5-2.0 zum Einreiben: Zum Schlusse der Cur, etwa zebn Tage vor dem Austritt, nehmen die Patienten Jodkali 1.0-3.0 pro die, 
ein Branch, der bei sämmtlichen Quecksilbercuren als Unterstützungscur geübt wird und somit für die Beurtheilung der Wirkung und des Werthes der einzelnen Mittel wohl mit Recht in Wegfall kommt.

Die kleinste Zahl von Inunctionen einer Cur betrug 10,- die grösste 50; in den meisten Fällen wurden 20-30 Einreibungen gemacht. Zeissl gibt 12-16 als Minimum, 30 als Maximum an, Müller 25 als Minimum, 35 als Mittel und 50 als Maximum.

Seit Januar 1880 bis Januar 1889 (oxclusive) wurden 303 vollständige Inunctionscuren geleitet und die betreffenden Individuen als geheilt, respective temporär geheilt entlassen. Unter diesen 303 Fällen sind solche Patienten natürlich nicht eingerechnet, welche keine vollständige Cur aus irgend einem Grunde durchmachen konnten, oder schon ausserhalb des Spitales eine theilweise antiluetische Cur gebraucht hatten; dasselbe gilt auch für die Besprechung der folgenden Quecksilberpräparate. Dagegen wurden zu den Inunctionen noch diejenigen wenigen Fälle gezählt, wo neben den Einreibungen einige Injectionen mit einem der Präparate aus irgend welchem Grunde - meist wurden die Injectionen schlecht vertragen und deshalb Inunctionen angewandt gegeben wurden; war das Verhältniss der Injectionen zu den Inunctionen ein grösseres, so dass man nicht bestimmen konnte, welcher Therapie als Haupteur die Heilung zuzuschreiben war, so wurden diese Fälle als gemischte angeführt.

Von den 303 als geheilt entlassenen Pationten kamen 26 wieder auf die Abtheilung mit Recidiven, um einer neuen Cur unterzogen zu werden.

Ist hier und da ein Fall zufällig bekannt geworden, wo sich ein früherer Patient einem Privatarzte oder in der Poliklinik mit Recidiv stellte, so wurde dies natürlich nicht in Berechnung gezogen, da sich sozusagen dieser Verlust an Recidiven für die Abtheilung auf alle angewendeten Mittel im Grossen und Ganzen gleichmässig erstreckt, und es ja ein merkwürdiges Spiel des Zufalls wäro, wenn ganz besonders bei dem einen oder anderen Mittel die Recidive für die Beobachtung auf der Abtheilung verloren gegangen wären. Im Uebrigen ist gerade die hiesige Klinik wegen ihres grösstentheils stationären Krankenmaterials (Prostituirte) 
besonders geeignet zur Beobachtung der Recidive. Dieselben betragen also nach don Inunctionscuren 9 Procent.

Von den vielen löslichen Präparaten, die man bei der Einführung der subcutanen Injectionen zuerst anwandte, kamen hier das Hydrargyrum peptonatum und formamidatum und das Sublimat. zur Verwendung.

Einer besonderen Beliebtheit hatte sich das erstere zu erfreuen, das schon 1877 auf der hiesigen Abtheilung eingeführt wurde und bis gegen Ende 1883 zur Verwendung kam, und zwar seit 1880 an nahezu 500 Kranken.

Das Quecksilberpepton verdankt seine Entstehung den Versuchen v. Bamberger's und speciell der Thatsache, dass die Injectionen mit einfachem Sublimat nach $L e w i n$ an der Injectionsstelle starke irritirende Wirkung ausübten, wofür sich die Erklärung in der Fällung der Eiweisslösungen im Unterhautbindegewebe durch das injicirte Sublimat fand. Um dies zu verhüten, entstanden eine Reihe von Präparaten, welche Eiweisslösungen nicht fällten und deshalb neben geringeren örtlichen Beschwerden auch eine rasche und sichere Wirkung erzielen sollten, so auch das Quecksilberalbuminat, das v. Bamberger angab, das zwar den Vortheil hatte, an der Injectionsstelle keine Reaction zu erzeugen und nicht schmerzhaft zu wirken, aber bald den bedenklichen Uebelstand aufwies, welcher der allgemeinen Verbreitung des Mittels von vorneherein entgegentrat, nämlich die Schwierigkeit seiner Bereitung, die geringe Haltbarkeit und das Auftreten von Reactionsknoten, die man ja doch hatte vermeiden wollen, sobald eine nicht ganz frisch bereitete Lösung in Anwendung kam. (Gschirhakl, Wiener med. Wochenschr. 1877.) So wurde denn das Albuminat von dem Autor selbst durch eine neue ähnliche Quecksilberverbindung.ersetzt, zu deren Herstellung statt des Albuminats die Peptone benutzt wurden; dieselben erleichtern die Herstellung und vermehren die Haltbarkeit des Präparates, da sie in Wasser leicht löslich sind, sich im Gegensatz zu den Eiweisslösungen leicht und klar filtriren lassen, nicht wie die letzteren die beständige Neigung zeigen, sich zu trüben und durch Hitze, Säuren, Alkalien nicht gefällt werden. (v. Bamberger, Wiener med. Wochenschr. 1876.)

Die Herstellıng des Präparates darf wohl als allgemein be- 
kannt vorausgesetzt werden. $1 \mathrm{Ccm}$. der Lösung entspricht 0.01 Sublimat, in welcher Menge anch die einzelnen Injectionen angewendet wurden.

Das nene Mittel fand bald nach seiner Veröffentlichung wegen seiner wenig irritirenden Wirkung Eingang in vielen Kliniken, um auf seinen therapeutischen Werth erprobt zu werden.

Von der hiesigon Klinik wurden in einer Dissertation (K. Zeitler 1880) 189 Fälle, die vom Januar 1878 bis Juni 1880 mit Pepton behandelt worden waren, aufs Genaneste beschrieben. Verfasser referirt zwar nicht über Recidive (die er grossentheils wegen der Kürze der Beobachtungszeit nicht hatte beobachten können) fand aber, was örtliche Reaction, Schmerz, Abscesse und Stomatitis betrifft, äusserst geringe Zahlen. Die sehr geringe allgemeine Reaction bestätigen auch Zeissl und Neumann. Rotter (Jahresber. d. ges. Med. II. 1879), der das Präparat in 11 Fällen anwandte, kam zn der Meinung, dass dasselbo schneller als alle übrigen, damals bekannten Qnecksilberverbindungen gegen Lues wirke und an der Kraft der Wirkung denselben nicht hintanstehe; freilich hatte er auch einige Misserfolge zu verzeichnen; als Maximum der Injectionen gibt er die geringe Zahl 13-15 an, Phlegmone und Gangrän schienen ihm im Ganzen selten aufzutreten. Ein ebenso günstiges Resultat veröfentlicht Martinea u (ebenda II, 1881), der in 172 Fällen Ammonpeptonquecksilber, ein mit dem Peptonquecksilber gleichwerthiges Präparat anwandte; die Wirkung dieses Mittels beschreibt er als rascheste und energischeste aller Behandlungsmethoden und würde sogar der Verlauf der Schwangerschaft begünstigt, so dass gesunde Kinder geboren würden. An den Injectionsstellen fand anch er keine krankhaften Veränderungen, selbst bei grossen Dosen nur selten Schmerz und nie Salivation, wenn nicht schon vorher ein Reizzustand der Schleimhant des Mundes und des Zahnfleisches vorbanden war. Uebereinstimmend mit Rotter und Martineau fand Pauer (J. D. Würzburg 1886, vom Münchener Krankenh.) rasche, besonders für schwere Formen günstige Wirkung, doch beobachtete er mehrere Infiltrationen und gegenüber andern Beobachtern ziemlich viele Salivationen (44 Procent). Oberländer (Jahresber. d. ges. Med. II. 1878) fand zwar nur 15 Procent Sa- 
livation und nicht nach jeder Injection Infiltrate, jedoch im Verlaufe von 1 bis 3 Monaten 62 Procent Recidive.

Bockhart (Monatsh. f. prakt. Derm., Bd. IV, Nr, 5. 1885) fand dagegen wieder häufige Schmerzen nach den Injectionen und Recidive im Durchschnitte vach 6 Monaten, während Mundaffectionen gering waren.

Diese wenigen literarischen Notizen genügen, um von den Resultaten, die mit dem Peptonquecksilber erzielt wurden, ein Bild zu bekommen. Sind es im Allgemeinen, was die Begleiterscheinungen dieser Therapie betrifft, auch günstige Resultate, ist die Wirkung auch von Verschiedenen als besonders rasch und energisch beschrieben, so wird der Werth derselben doch bedeutend verringert durch das häufige Auftreten von Recidiven, wie dies Oberländer und Bockhart constatirten.

Auf der hiesigen Abtheilung wurden in den Jahren 1880 bis 1883435 Patienten ausschliesslich mit subcutanen Injectionen von Quecksilberpepton behandelt und als geheilt entlassen. Das Minimum der Injectionen beträgt 10, das Maximum 30. $259 \mathrm{~Pa}$ tienten erhielten 25 Injectionen, 142 weniger, 34 mehr wie 25. Die Injectionen wurden täglich gemacht, pro dosi 0.01 Sublimat und zwar subcutan rechts und links dicht neben der Wirbelsäule unterhalb des unteren Schulterblattwinkels, welche Stelle man wegen der geringen Schmerzhaftigkeit als die geeignetste fand. Eine Nacheur mit Jodkali wurde in beinahe allen Fällen angewendet. $\nabla$ on diesen 435 geheilten Syphilitischen kamen 69 , also 16 Procent Recidive wieder zur Behandlung in der Klinik, darunter 8 zu mehrmaliger Cur. Die meisten Patienten kamen schon nach 3 bis 7 Monaten wieder mit Recidiven; 42 Pationten hatten aus irgend wolchen Gründen keine vollständige Injectionscur bekommen und mit Inunctionen die Cur vollendet, von diesen kamen 8 wieder mit Recidiven.

An die Stelle des Quecksilberpeptons trat hier Ende 1883 in der Syphilisbehandlung das Quecksilberformamid, ein Präparat, das kurz zuvor von Liebreich angegeben wurde. Die Versuche, die zuletzt zu diesem Präparate führten, beruhten auf der Idee, eine Quecksilberverbindung zu finden, welche die Albuminate des Körpers nicht angreift und es zugleich gestattet, in einem gegebenen Momente eine grössere Menge von Quecksilber im Körper 
gleichmässig anzuhäufen. Dadurch, dass das Formamidquecksilber keine Verbindung mit dem Körper-Eiweiss eingeht, glaubte Liebreich, könne es sich gleichmässig im Organismus verbreiten, da ja nirgends ein Verbindungswiderstand eintrete, und beginne dann die Zerlegung des Präparates, so werde gleichmässig an Ort und Stelle, wo Molecül für Molecül sich zerlegt, eine grössere Menge von Quecksilber abgeschieden, und dadurch sei die Kraft und die Einwirkung desselben auf das syphilitische Gift bedeutend erhöht. Durch diese physiologisch-chemische Wirkung sei das Formamid im Stande, leichter und schneller sich zu verbreiten, als namentlich das Sublimat und dementsprechend der Erfolg bei seiner Anwendung ein besserer.

Das Präparat selbst $\underset{\mathrm{CO}}{\mathrm{H}} \cdot(\mathrm{NH}) \mathrm{Hg}$ ontsteht aus $\underset{\mathrm{CO}}{\mathrm{H}}$ durch Behandlung von $\mathrm{HgO}$ mit Formamid. Es wird eine 1procentige Lösung angewendet und davon $1 / 2-1$ Spritze injicirt; $1 \mathrm{Ccm}$. enthält $0.01 \mathrm{Hg} 0$. Die Anzahl der Injectionen soll 30 nicht überschreiten.

Seinen theoretischen Auseinandersetzungen entsprechend, will Liebreich selbst eine gute Wirkung seines Mittels gesehen haben; schon nach einer Injection habe man eine Wirkung constatiren können. Er gibt ferner an, dass nach Anwendung des Formamids die Recidive bedentend vermindert würden und selten Salivation entstehe.

Dem gegenüber stehen die Resultate, welche nach Erprobung des Quecksilberformamids von Verschiedenen publicirt wurden.

In der hiesigen Klinik beobachtete und beschrieb Fr. Schmitt in einer Dissertation (1884) 51 Fälle und konnte einen wesentlichen Vorzug gegenüber dem Quecksilberpepton nicht constatiren; ebensowenig fand Lassar einen Vorzug vor dem ChlornatriumSublimat. Kopp sagt in seiner Abhandlung über die Behandlung der Syphilis mit subcutanen Injectionen von Quecksilberformamid (Vierteljahresschr. f. Derm. u. Syph. 1885), dass er in der That in einer grossen Anzahl von Fällen einen curativen Effect sah, dass diese Fälle aber sämmtlich den leichteren Formen angehören, die als maculöse und papulöse Exantheme, Plaques muqueuses oder nässende Papeln dem Beginne der Secundärperiode entsprechen und häufig unter rein localer Behandlung zur Involution gelangen. 
Er glaubt daher diesen günstigen Resultaten keinen allzugrossen Werth für die therapentische Beurtheilung des Formamids beilegen zu dürfen, umsomehr als grosspapulöse Syphilide und derbere Infiltrate sich als sehr resistent gegen die eingeschlagene Therapie erwiesen, und schiebt die Ursache dieser wenig intensiven Einwirkung des Präparates auf den syphilitischen Process der raschen Ausscheidung desselben aus dem Organismus zu. Dies fand er auch durch die chemische Untersuchung des Harnes und der Fäces bestätigt, wonach das Quecksilber den Organismus so rasch verlässt, dass es zu einer Anhäufung des Medicamentes gar nicht kommt, so dass die Einwirkung desselben auf das syphilitische Virus nicht genügend lang und intensiv erscheint, um dasselbe zu vernichten. Auch was die Häufigkeit der Recidive betrifft, räumt Kopp dem Liebreich'schen Präparat keinen Vorzug ein, da ihm im Gegentheil dieselben nach dieser Behandlung äusserst häufig zu sein scheinen. Ebensowenig glaubt Müller in einer Beilage zur medicinischen Wochenschrift, dass das Formamid dem Sublimat vorzuziehen sei, was auch in einer Statistik aus dem Münchener Krankenhaus 1. d. Isar von Pauer bestätigt wird, der fand, dass bei Sublimatinjectionen 71 Procent Heilungen, 27 Procent Besserungen vorkamen und nur 2 Procent erfolglos behandelt wurden, wäbrend bei Quecksilberformamid 59 Procent Heilungen, 32 Procent Besserungen eintraten und 9 Procent erfolglos blieben. Aehnlich spricht sich Zeiss1 aus (Jahrebber. d. ges. Med., II., 1882). Er sagt, dass sich das Formamid durchaus nicht von anderen Quecksilberpräparaten unterscheidet, $d_{\mathrm{w}}$ es die Symptome in gleicher Zeit zum Schwinden bringt und weder Salivation, noch ebenso häufige Recidive verhindern kann. Das ungünstigste Resultat betreffs der Recidive fand Bockhardt (Monatsh. f. prakt. Derm. 1885, II., Nr. 5), der das Formamid bei 28 Patienten anwandte, von denen kein einziger von Recidiven verschont blieb; das früheste Recidiv trat in 9 Wochen, das späteste in 4 Monaten anf, und zwar hatten 2 Patienten 1 Recidiv, $16: 2,9: 3$, einer 4 bekommen. Jedoch für leichtere Formen hält Bockhardt das Formamidquecksilber für das am raschesten wirkende Mittel.

Ganz entsprechend diesen therapeutischen Resultaten fand sich hier das Verhältniss der Recidive nach der Formamidbehand- 
lung sowohl den Inunctionscuren als auch den Sublimatinjectionen gegenüber, welch letztere Therapie ja nach Liebreich besonders vom Quecksilberformamid übertroffen werden soll. Es fanden sich 89 Fälle, welche ausschliesslich mit dem Präparate behandelt wurden und als geheilt entlassen werden konnten. Die meisten der Patienten hatten zwischen 20 und 30 Injectionen erhalten und zum Schlusse der Cur analog den anderen Behandlungsmethoden Jodkali, täglich $1 \cdot 0-3 \cdot 0$, bekommen. Speciell für die Therapie mit Formamid ist von Lassar eine vollständige Nebenbehandlung mit Jodkali namentlich bei schweren Formen empfohlen worden, gegen welche combinirte Behandlung sich jedoch Liebreich aus chemischen Gründen aussprach. In den Journalen und Krankengesehichten sind wenigstens keine üblen Folgen durch diese Nachbehandlung mit Jodkali bei Anwendung des Formamids verzeichnet.

Auf diese 89 Fälle treffen.16 Procent Recidive, die durchschnittlich etwa innerhalb eines halben Jahres wieder auf die Abtheilung kamen, mit Ausuahme von 2 Patientinnen, die sich erst nach 3 Jahren wieder mit Recidiven zur Behandlung einstellten, von denen die eine jedoch schon vorher anderwärts wegen Recidiv in Behandlung war, die andere keine zuverlässigen Angaben machte. 34 Patienten waren theils mit Injectionen, theils mit Inunctionen behandelt worden und treffen auf dieselben 8 Recidive. Stellen wir diese 16 Procent Recidive nach Behandlung mit Pepton und Formamid den Inunctionen gegenüber, die nur 9 Procent aufweisen, so kommen wir zu einem Resultate, das heutzutage auch von der Mehrzahl der Aerzte und Forscher anerkannt ist, dass diese leicht löslichen und schnell in die Blutbahn gelangenden Präparate, mögen sie auch mehrere praktische Vortheile besitzen, lange nicht der Wirkung der graten Salbe gleichkommen.

Wohl keines aller zur Syphilistherapie angegebenen Quecksilbersalze hatte so viele Kämpfe um seine Existenz durchzumachen, als das Sublimat; der Grund lag in dem Auftreten jener üblen Nebenerscheinungen, die man gerade immer bemüht war, ans der Injectionstherapie zu bannen, und so knüpft sich an die Syphilisbehandlung mit Sublimat ein gut Theil Geschichte der Syphilistherapie der Neuzeit. 
Es ist begreiflich, dass man, als die Injectionstherapie im Entstehen war, jenes Quecksilbersalz am meisten verwendete, das $\mathrm{ja}$ in so vielen anderen Beziehungen Verwendung in der allgemeinen Therapie fand, das Sublimat, Einen zähen Vertheidiger hat es in Lewin gefunden, der es ausschliesslich verwendete und anch die besten Erfolge damit erzielte. Er gebrauchte eine 1procentige Lösung und behandelte von 1865 an gegen 14000 Kranke auf seiner Abtheilung in Berlin mit durchschnittlich 25 Injectionen (Berliner klin. Wochenschr. 1876, Nr. 45), Nach langjähriger Erprobung des Mittels kam or zu dem Schlusse, der in der Vierteljahresschr. f. Derm. u. Syph. 1883 mitgetheilt ist, dass die Recidive seit Einführung der Sublimatinjectionen in Bezug auf Quantität entschieden geringer, in Bezug auf Qualität entschieden weniger bösartig geworden seien. Ja er beobachtete sogar eine Abnahme der Recidive von 80 Procent auf 40 Procent, gegenüber den früheren Behandlungsmethoden, wie er schon 1876 in der Berliner klin. Wochenschr. mittheilt, und gibt als besten Beweis für die Qualität seiner Methode an, dass schon seit langer Zeit die schwersten Formen allgemeiner syphilitischer Erkrankungen auf der Abtheilung unter den Prostituirten nicht mehr vorkämen. Jedoch viele andere Forscher, die fast gleichzeitig Versuche mit dem Sublimat anstellten, fanden alle heftige entzündliche Reactionen an der Einstichstelle, wobei durch die Verbindung des Sublimats mit dem Eiweiss des Unterhautbindegewebes ein guter Theil Injectionsflüssigkeit für die Bekämpfung der Erkrankung verloren ging, ferner bedentende Schmerzen, so dass viele Kranke die Fortsetzung der Cur weigerten (Gschirhakl, Wiener med. Wochenschr. 1877). Auch v. Bamberger wendet sich gegen die Sublimatinjectionen und zwar wegen der häufigen Abscessbildung in der Haut und der heftigen Schmerzen (ebenda 1876). Dagegen hatte Lewin bei jenen 14000 Fällen nur 20 Abscesse zu verzeichnen, während er oft Schmerzen beobachtete. Man versuchte nun auf verschiedenem Wege diesen Nachtheil der Sublimatinjectionen zu beseitigen, indem man einestheils solche Präparate anwendete, die von vorneherein keine Fällung des Körper-Eiweisses zu Stande kommen lassen, wie das Quecksilberpepton und Formamid, anderntheils die Lösung des im Körper gebildeten und namentlich zu starken Retentionsknoten führenden Sublimatalbu- 
minats durch Hinzufügen von Kochsalz zu beschleunigen suchte, um die irritirende Wirkung des reinen Sublimats und dadurch die localen Nachtheile zu vermeiden. Dem Schmerz suchte man nach Liégois durch Hinzusetzen von kleinen Quantitäten von Morphium zur Injectionsflüssigkeit entgegenzutreten, nach Decker durch vorhergehende Injection von Cocaïn (Münchener med. Wochenschr. 1887, Nr. 39). Gegen die angeblichen Vortheile der von Müller und Stern angegebenen Lösung des Doppelsalzes Quecksilberchlorid-Chlornatrium, bestehend aus 1 Theil Sublimat, 10 Theilen Chlornatrium, 250 Theilen Wasser (Jahresber. d. ges. Med., 1878, II.) wendet sich Liebreich (Vierteljahresschr. f. Derm. u. Syph. 1887), indem er bemerkt, dass, wenn auch Sublimat mit Chlornatrium eine Doppelverbindung eingeht, doch die Existenz dieser Verbindung in Lösung nicht wahrscheinlich sei, weshalb bei der subcutanen Injection das Chlornatrium schneller als das Sublimat resorbirt und dadurch der Nutzen der gleichzeitigen Injection bedeutend abgeschwächt wird. Liebreich glaubt deshalb, dass es für die angestrebte bossere Löslichkeit und Resorbirbarkeit des Sublimats viel vortheilhafter sei, nach Hinrich Sublimat und Kochsalz, und zwar letzteres in relativ grossen Quantitäten, innerlich anzuwenden. Was das Quantum Kochsalz für die subcutane Injection betrifft, so wandte Gschirhakl bei $100^{\circ} 0$ einer 1procentigen Sublimatlösung 6.0 Chlornatrium an (Wiener med. Wochenschr. 1877). Kratschmer veröffentlicht (ebenda 1876) Versuche, nach denen erst, wenn die Sublimatlösung 4-5 Procont Chlornatrium enthält, keine Fällung und Trübung von Eiweisslösungen und transsudirtem Serum eintreten, and eine 10 Procent Chlornatrium enthaltende Sublimatlösung noch keine Reizerscheinungen bei der subcutanen Injection liefert.

In nenester Zeit wurde wiederum die reine Sublimatlösung versucht von Bergmann in Moskau (Wiener med. Wochenschr. 1889, Nr. 14) und zwar in etwas geringerer Dosirung für die Injection. Er gab 200 Patienten täglich 0.0075 Sublimat im Durchschnitt mit 26-28 Injectionen und fand, dass die Schmerzen nicht stärker, aber kürzer dauernd seien, als nach Injectionen mit unlöslichen Präparaten, dass Abscesse nie vorkamen und Mundschleimhautaffectionen nicht erheblich waren; da ihm auch die Zahl der Recidive nach dieser Methode eine geringere zu sein 
scheint, so gibt er dieser wässerigen Sublimatlösung vor anderen, insbesondere den unlöslichen Präparaten den Vorzng.

Auf der hiesigen Abtheilung kam das QuecksilberchloridChlornatrium in folgender Zusammenstellung zur Anwendung:

Rp. Hydrarg. bichlorat. corros. $0 \cdot 1$

Nati'. chlorat. $1 \cdot 0$

Aq. destill. $10 \cdot 0$

filtra. D. S. zur Injection.

Diese Lösung ist also eine 1procentige Sublimatlösung mit 10 Procent Kochsalz und bekamen die Patienten mit einer ganzen Pravaz'schen Spritze jedesmal 0.01 Sublimat. Im Ganzen konnten 89 Patienten, die mit diesem Mittel behandelt wurden, als geheilt entlassen werden; davon hatten 7 eine grössere Anzahl von Inunctionen erhalten, von denselben wurden 2 Recidive beobachtet. Die Zahl der Inunctionen schwankt zwischen 10 and 39, die Durchschnittszahl beträgt 23. Ein 8 Monate altes Kind bekam 24 Injectionen zu 0.005 . Eine Nachcur von geringen Dosen mit Jodkali fand bei allen Patienten statt. Von den 82 mit Sublimat behandelten Kranken kehrten mit Recidiven auf die Abtheilung zurück 13 Procent; ein Resultat, das den Werth dieser Therapie gegenüber den bisher genannten Quecksilberverbindungen als den Inunctionen am nächsten stehend erscheinen lässt und auch mit dem Urtheile vieler Praktiker und Forscher übereinstimmt. Diese günstige Wirkung der Sublimatinjectionen wird erklärt durch die Bildung von Sublimateiweiss unter der Haut, das sich nur allmälig löst und in Circulation gebracht wird (Bockhart, Monatsh. f. prakt. Derm. 1885, IV. Bd., Nr. 5), was man früher gerade für nachtheilig hielt und durch Pepton- und Formamidverbindung zu verhindern suchte.

Nachdem nun die therapentisclen Erfolge, die mit den leichtlöslichen Vitteln im Allgemeinen den an sie gestellten Erwartungen nur wenig entsprachen, mit Ausnabme jedoch der Sublimatinjectionen, deren verhältnissmässig günstige Erfolge ja nicht auf der Löslichkeit, sondern auf der oben beschriebenen Eigenschaft des Sublimats beruhen, versuchte man die Auwendung von unlöslichen und schwerlöslichen Präparaten, um die allzuschnelle Ausscheidung des Quecksilbers aus dem Körper zu vermeiden und eine langsamere, allmäligere Einwirkung auf das syphilitische Gift zu er- 
zielen, zumal man durch vielfache Versuche über die Ausscheidung des Quecksilbers aus dem Körper zu der Ansicht gelangte, dass die eclatante Wirkung der Einreibungen mit grauer Salbe gerade dem Factor zu verdanken sei, dass der Organismus bei langsamer Ansscheidung des Quecksilbers sehr lange unter dem Einflusse desselben gebalten werde.

Voran ging Scarenzio mit der Einführung der Calomelinjectionen, die anch auf der hiesigen Abtheilung eine Zeit lang (1875/76 bis 1877/78) zur Verwendung kamen, aber nicht mehr in den Bereich dieser Arbeit gehören. Die Erfolge, die man hier mit Calomel erzielte, hat Kölliker (J. D. 1877) genan beschrieben. Mit der Zeit mehrten sich die Berichte über diese Therapie, so dass jetzt die Behandlung mit Calomelinjectionen bezüglich der therapentischen Wirkung in den Ruf der besten subcutanen Applicationsmethode gekommen ist und von vielen bedeutenden Forschern ihr eine Stelle unter den verschiedenen Syphilistherapien zu Theil wurde, die den Inunctionen am nächsten kommt oder gar denselben gleichwerthig ist. Und doch zeigen die Injectionen mit Calomel wieder Nachtheile, die ihren Ruf bedentend schwächen, nämlich häufige Abscessbildung und oft sehr heftige Schmerzen an der Einstichstelle. Kein Wunder also, dass in der Folge eine Reihe von neven Präparaten entstand, die wegen ibrer schweren Löslichkeit die Erwartung rechtfertigten, der Wirkung des Calomel gleichzukommen und doch die örtliche Reaction vermeiden sollten. E's sind dies vor Allem die Oxyd- und Oxydulsalze, von denen v. Watraszewsky in den Monatsh. f. prakt. Derm. 1887, Nr. 22, eine Zusammenstellung gegeben hat; derselbe findet ihre Anwendung erfolgreich und lässt ihre Wirknng abhängen von ihrem Procentgehalt, ebenso den Grad der Iocalen Reaction von der Concentration des Präparates.

Von diesen unlöslichen Quecksilberoxyden und Oxydulen kamen hier zur Anwendung das Hydrargyrum oxydulatum tannicum und das Hydrargyrum oxydatum carbolicum. Das erstere Präparat ist von seinem Autor vorzugsweise für innere Verabreichung bestimmt worden, da die bisher für diesen Zweck empfoblenen Mittel (Calomel, Sublimat, Protojoduret etc.) störende Nebenerscheinungen des Intestinaltractus hervorriefen, wie Gastralgien, Kolikschmerzen und bäufige Durchfälle, wodurch ja auch 
der Kräftezustand der Patienten oft hart angegriffen und die genügende Resorption des Medicamentes bedeutend fraglich wird. Neben einer vortrefflichen Haltbarkeit hat das Hydrargyrum tannicum, ein dunkelgrünes, geschmack- und geruchloses Pulver, vermöge seiner chomischen Eigenschaften am meisten von allen intern angewendeten Quecksilberverbindungen Aussicht, vom Verdaunngstractus gut vertragen zu werden. Es wird von verdünnter Salzsäure nicht angegriffen, wohl aber von Alkalien, wenn auch in ganz verdünntem Zustande, und scheidet sodann einen aus ganz feinen Quecksilbertheilchen bestehenden Schlamm aus. Diese Reduction des Präparates geht, wie Lustgarten (Wiener med. Wochenschr. 1884, Nr. 11-14) angibt, im Darm durch kohlensaure Alkalien vor sich, während das Mittel bei normaler Magenverdauung keine bedeutende Veränderung erleidet. Wie vollends die resultirenden Quecksilbertheilchen zur Resorption gelangen können, erklärt Lustgarten (ebenda) auf verschiedene Weise.

Lustgarten wendete 0.3 pro die (mit Milchzucker in Oblaten) an und fand seinen Erwartungen gemäss, dass das Mittel vom Verdauungstractus gut vertragen werde. Ebenfalls günstige Resultate fand Dornig (Wiener med. Wochenschr. 1885, Nr. 27). Er gab das Medicament in Dosen von $0 \cdot 1$, täglich 0.3 und beobachtete einerseits, dass es gut vertragen werde und es niemals zu Koliken oder Durchfällen kam, andererseits im Allgemeinen auffallend rasches Zurückgehen der Exantheme der Secundärperiode.

Dagegen will Pauly (Berliner klin. Wochenschr. 1884, Nr. 47) beftige Störungen des Verdauungstractus beobachtet haben, obwohl er die Dosis 0.3 pro die nie überschritt. Lesser (Lehrb. der Haut- u. Geschlechtskrh.) empfiehlt das Hydrarg. oxydul. tannicum als ein Mittel, das an Wirksamkeit alle anderen internen Quecksilbermittel übertrifft, da es wegen seiner besseren Leichtverträglichkeit in sehr viel grösseren Dosen gegeben werden kann; er konnte selbst nach einer ohne Verordnung genommenen Dosis von 0.45 keine nachfolgenden Störungen constatiren. Besonders empfiehlt er, das Mittel in Pillenform zu geben mit der Tagesdosis 0.3 .

Auf der hiesigen Abtheilung wurde das Präparat ebenfalls in Pillenform angewandt; und zwar ordinirt: 
Rp. Hydrarg. oxydul. tannic. 0.05

Pulv, et Extract. Liquirit. q. s.

ut f. pil. D. tal. dos. Nr. 100, S. 3mal tgl. 2 Pillen.

Die Zahl der damit behandelten Patienten beträgt 33. Beinahe bei Allen finde ich in den Krankengeschichten die Notiz: Opiumtinctur gegen starke diarrhoische Stühle verzeichnet; eine Patientin hatte Nachts 6mal diarrhoischen Stuhl, nachdem sie Tags vorher 6 Pillen $=0.3$ Hydr. oxyd. tann. genommen hatte. Je nach der Schwere der Fälle richtet sich ratürlich die Anzahl der gegebenen Pillen, sie schwankt zwischen 60 und 306 und beträgt im Durchschnitt etwa 160.

Von diesen 33 als geheilt entlassenen Patienten stellten sich $6=18$ Procent wieder mit Recidiven ein, und zwar schon nach 2-7 Monaten, durchschnittlich in 5 Monaten. Dieser verbältnissmässig ungünstige Procentsatz der Recidive ist nur dadurch zu erklären, dass durch die häufigen Störungen des Intestinaltractus die Resorption des Quecksilberoxyduls bedeutend vermindert wurde, wodurch eine nachhaltige Wirkung des Quecksilbers im Organismus nicht hat stattfinden können. Mag auch das Hydr. oxyd. tann. die Sehleimhant des Magens und Darmtractus nicht in so hohem Grade angreifen, wie die anderen zu innerem Gebrauche angegebenen Mittel und denselben vorzuziehen sein in Fällen, wo man aus gesellschaftlichẹn Gründen weder die Inunctionscur, noch Injectionen anwenden kann, so ist es doch hinsichtlich seiner Wirkung den bis jetzt hier angeführten Injectionstherapien nicht gleich zu setzen, geschweige denn einer energischen Inunctionscur.

Auch das Hydrargyrum oxydatum carbolicum wurde zuerst in Pillenform angewandt, und zwar von Gamberini (Rivista italiana di terapia e igiene 1886, Nr. 3). Derselbe hoffte durch die Verwendung zweier antiparasitärer Mittel dem syphilitischen Virus am erfolgreichsten entgegentreten zu können, eine Ansicht, welche die Entstehung von noch mehreren Quecksilberpräparaten anregte. Das Hydr, oxyd. carbol., das sich in seiner chemischen Formel folgendermassen darstellt:

$$
\begin{array}{lll}
\mathrm{C}_{6} & \mathrm{H}_{5} & 0 \\
\mathrm{C}_{6} & \mathrm{H}_{3} & 0
\end{array}>\mathrm{Hg}+\mathrm{H}_{2} \mathrm{O}
$$

entsteht durch Fällung einer verdünnten, wässerigen Sublimatlösung mit einer concentrirten von Kaliumearbonat freien alkoholischen 
Phenolkalilösung. Dฮ̄ erhaltene Niederschlag wird nach längerem Stehen, nach Befreiung von Chlor über $\mathrm{H}_{2} \mathrm{SO}_{4}$ getrocknet. Gamberini hatte mit dem Präparate, namentlich bei seiner Verabreichung in Pillenform, günstige Resultate erzielt. Ebenso Śzadek (Monatsh. f. prakt. Derm. 1887, Nr. 5 u. 8), der nach seinen Erfolgen mit dem Mittel in Pillenform auch die Application mit subcutanen Injectionen versuchte. Die Injectionen waren nur unbedeutend schmerzhaft und gaben Knoten und Indurationen an der Einstichstelle, jedoch keine Abscesse. In mehreren Fïllen kam es zu leichter Stomatitis. Zu vollständigem Heilerfolg genügten 16-18 Injectionen in frischen Fällen. Auf der hiesigen Abtheilung wurde das Präparat theils in Pillenform, theils zu subcutanen Injectionen benutzt, und zwar ordinirt:

Rp. Hydrarg. oxydat. carbol. $0 \cdot 6$

Extr. Opii $0 \cdot 3$

Extr. et pulv. Liquirit. q. s.

ut f. pil. Nr. 30. Ds. tgl. 4 Pillen.

Rp. Hydrarg. oxydat. carbol. $0 \cdot 5$

Mucil. Gumm. arab. $1 \cdot 0$

Aq. dest. $25 \cdot 0$

MDS. zur Injection.

Die Anzahl der verabreichten Pillen schwankt zwischen 60 und 140. Einige Patienten hatten Pillen und Injectionen erhalten. Von Injectionen, welche alle 2 Tage gemacht wurden, waren 15-20 nöthig, nur einmal 25, um die syphilitischen Erscheinungen ganz zur Involution zu bringen. Was die Nebenerscheinungen dieser Therapie betrifft, so traten zwar bei der internen Anwendung häufig mehr oder minder starke Leibschmerzen und Diarrhöen auf, doch führte die örtliche Reaction an der Einstichstelle der Injectionen nie zu Abscess, sondern nur zu kleineren und grösseren Knoten, die in der Regel nur leichte, in einigen Fällen aber sehr intensive Schmerzen verursachten. Leichtere Stomatitiden kamen öfters, schwerere nur zweimal zur Beobachtung. Auch Störungen von Seiten des Intestinaltractus traten in mehreren Fällen nach einer grösseren Anzahl von Injectionen anf und einmal bei einer 20jährigen Patientin nach der siebenten Injection ein Shock-ähnlicher Zustand (Decker, Münchner med. Wochenschr. 
1888, Nr. 5), ebenso nervöse Erscheinungen, wie sie auch $\nabla$. Watraszewski (Monatsh. f. prakt. Derm. 1887, Nr. 22) beobachtet hatte. Die einzelnen Fälle wurden näher beschrieben von Happel (J. D. 1888). Die Verhältnisszahl der beobachteten Recidive beläuft sich noch böher, als bei dem Hydrarg. oxydul. tannic., es kamen nämlich von den 22 behandelten und als geheilt entlassenen Patienten $6=27$ Procent mit Recidiven wieder zur Beobachtung, und zwar nach kurzer Zeit, im Durchschnitte in 4 bis 5 Monaten. Die Wirkung des Präparates war also auf keinen Fall, obgleich es eine unlösliche Quecksilberverbindung ist, eine starke und lang anhaltende, und dürfte dasselbe in Folge dessen wegen der relativen Häufigkeit der Recidive vielleicht nur bei leichteren Formen zur Anwendung gelangen, sicherlich aber niomals eine bevorzugtere Stellung in der Syphilistherapie einnehmen.

Ferner wurden hier mit einem neuen Präparate, dem Hydrargyrum salicylicum, Versuche gemacht, das im Jahre 1887 in weiteren Kreisen bekannt wurde. Im Jahre 1886 wurde es von Veiga und Delgado hergestellt, durch Fällung einer wässerigen Lösung von salpetersaurem Quecksilberoxyd mittelst einer alkoholischen Lösung von salicylsaurem Alkali; es resultirte ein amorphes, weisses, geruch- und geschmackloses Pulver, das neutral reagirt und in Wasser und Alkohol schwer, in Glycerin leicht löslich ist, mit der chemischen Zusammensetzung:

$$
\mathrm{C}_{6} \mathrm{H}_{4}<\mathrm{COO}
$$

und einom constanten Gehalte vou 59 Procent Quecksilber. Im thierischen Organismus wird es trotz seiner schweren Löslichkeit in Wasser gelöst, wahrscheinlich durch das überall im Gewebe vorhandene Chlornatrium. Bezüglich seiner allgemeinen therapentischen Wirkung fanden Ellenberger und Hofmeister (Therapeut. Monatsh. II., 8., 1888), die es nach ihren Versuchen für ein vorzügliches antiseptisches, anticymotisches und desinficirendes Mittel halten, dass eine Wirkung schon in der Verdünnung I : 20.000 hervortritt. Auf der anderen Seite constatirt Müller (Monatsh. f. prakt. Derm. VIIT., Bd., Nr. 7), dass es die Giftigkeit der anderen Quecksilberverbindungen nicht besitzt und deshalb grosse Dosen vertragen werden, wie Versuche an diesen be- 
wiesen. Aehulich, wie das Hydrarg. oxydat. carbol. verdankt das Präparat seine Einführung in die Syphilistherapie dem Gedanken, besonders wirksam gegen die Syphilis als parasitäre Krankheit einwirken zu können durch die Anwendung von zwei antiparasitären Mitteln.

Silva Araujo (in Rio de Janeiro) ging von dieser Ansicht aus und machte 1886 die ersten Versuche mit diesem Mittel, bei dessen innerer und subcutaner Anwendung er die besten Erfolge erzielt haben will. Neben rascher und energischer Wirkung soll es rom Intestinaltractus gut vertragen werden und selbst die letzten luetischen Erscheinungen mit Erfolg bekämpfen können. Unter 400 Fällen sah er nur ein einziges Mal unangenehme Folgen. Zu einem ebenso glänzenden Resultate gelangte auch Chaves (Medical and Surgical Reporter, Philadelphia 1887), der dem Präparate einen Platz in der Syphilistherapie einräumt, der dem Jodkali und Calomel gleichkommt. Besonders rühmt er das Mittel, da keine Nebenerscheinungen, keine Stomatitis auftreten, und es innerlich verabreicht, gut vertragen wird. Szadek (Monatsh. f. prakt. Derm. 1888, Nr, 10), der das Präparat in Form von Pillen und jntramusculären Injectionen anwandte, sah schon nach 3 bis 5 Tagen ein Zurückgehen der luetischen Erscheinungen, doch musste er bei einem hartnäckigen Falle zu Inunctionen schreiten und widerstand die Drüsenschwellung in allen Fällen, wie bei den anderen Mitteln. Affectionen des Magendarmeanals wurden nicht beobachtet, auch nicht bei innerer Verabreichung. Das letztere wurde jedoch von Schwimmer (Wiener med. Wochenschr. 1889, Nr. 8), der nenerdings Versuche mit Hydrarg. salicyl. in Pillen und Pulverform anstellte, nicht bestätigt, da er im Gegentheil stets so heftige Reizerscheinungen von Seiten des Verdauungstractus erfolgen sah, dass er das Präparat mit Laudanum verband, um die Toleranz des Schleimhauttractus gegen das Medicament zu erhöhen; ferner beobachtete or in sehr vielen Fällen Stomatitis. Nach vierwöchentlicher Behandlung gingen jedoch sowohl die Hautsyphilide als gleichzeitige Geschwürsprocesse der Schleimhäute zurück. Er hält deshalb das Quecksilbersalicylat für ein vorzügliches Antilueticum, namentlich als internes Mittel mit dem nämlichen Erfolge, wie die externen Behandlungsmethoden mit der grauen Salbe oder Calomel. Was jedoch das Auftreten 
von Recidiven betrifft, so constatirt Neumann (Wiener med. Wochenschr. 1888, Nr. 47), dass sie nicht minder rasch als bei anderen intern angewendeten Präparaten auftreten. Auch die Injectionsmethode wurde von Neumann versucht, und zwar intramusculär, in einer Dosirung von Hydrarg. salicyl. 0.5, 0l. amygd. 15.0. Die Application fand er schmerzlos und ohne örtliche Reaction, wie Infiltrate oder Abscesse, und die Wirkung als besonders rasch und energisch vermöge des hohen Quecksilbergehaltes. Bezüglich seiner Wirkung stellt er das Präparat in eine Reihe mit dom Sublimat und Peptonquecksilber, glaubt aber nicht, dass die Häufigkeit der Recidive bei der Anwendung dieser Quecksilberverbindung geringer würde.

Die auf der hiesigen Abtheilung bezüglich der Hauptsache, dem Heilerfolge, gemachten Erfahrungen stimmen mit den meisten dieser Veröffentlichungen gerade nicht überein, denn das Mittel reichte in der Mehrzahl der Fälle nicht aus, um die luetischen Erscheinungen vollständig zum Schwinden zu bringen, so dass bei den meisten Patienten noch Einreibungen als Nachcur eingeleitet werden mussten. Das Präparat, das zur Anwendung gelangte, hatte folgende Dosirung: Hydrarg. salicyl. 1·0, Ol. amygd. 10.0. Die Patienten erhielten Anfangs alle 8 Tage, bald darauf aber, da hiebei kein besonderer Erfolg zu erzielen war, alle Wochen zweimal je eine ganze Pravaz'sche Spritze $(=1 \cdot 0)$ und somit bei jeder Injection $0 \cdot 1$ Hydrarg. salicyl.

Einen grossen Vortheil hat das Präparat vor anderen Quecksilbersalzen, die za Injectionen verwendet werden, dass es nämlich keine örtliche Reaction im Gefolge hat; weder Schmerzen, höchstens geringen Grades bei der ersten Injection, noch Infiltrationen oder gar Abscesse konnten wahrgenommen werden. Störungen im Bereiche des. Intestinaltractus traten nie ein, jedoch verschiedene Male Stomatitis.

15 Patienten wurden mit Injectionen dieses Mittels behandelt; die Krankengeschichten derselben sind in der. InauguralDissertation von Fischer 1889 veröffentlicht. 14 konnten als geheilt entlassen werden nach durchschnittlich 9 Injectionen, die meisten jedoch erst, nachdem sie noch 5-7 Inunctionen als Nachcur bekommen hatten, bis die Symptome vollständig zurückgegangen waren. In einem Falle, wo ein stark entwickeltes Exanthem vor- 
lag, waren sogar noch 16 Inunctionen nöthig, weshalb dieser Fall zu den gemischten Behandlungsweisen gerechnet werden muss; derselbe kam übrigens nach 5 Monaten mit Recidive wieder. Von den übrigen Patienten kamen schon nach 3 Monaten zwei mit Recidiven zurück $=15$ Procent. Sehen wir davon ab, dass ein Urtheil über den Werth des Präparates bei dieser geringen Anzahl von Fällen und bei der meist eingeleiteten Nachcur mit Inunctionen absolut unsicher ist, so würde der Procentsatz der Recidive ungefähr den Resultaten von $\mathrm{Neumann}$ entsprechen, der die Wirkung des Salicylats der des Quecksilberpepton und Sublimat gleichschätzt. Auch stimmt unsere Beobachtung wenigstens in Betreff der Injectionen mit der Ansicht von Szadek, der das Quecksilbersalicylat bis jetat insofern für das beste Quecksilberpräparat hält, weil es trotz seiner schweren Löslichkeit bei innerer Anwendung und intramusculären Injectionen weder locale Reizerscheinungen, noch allgemeine Störungen hervorruft.

Nach dem Hydrarg. salicyl. wurde hier noch im Sommer und Herbst 1888 das Oleum cinereum angewandt.

Theils die relativ günstigen Resultate, die man mit den Quecksilbersalzen gegenüber der grauen Salbe machte, theils wohl die selbst erzielten guten Erfolge mit der alten Inunctionscur mochten Lang zu dem Gedanken gebracht haben, den er seinem neuen Präparate zu Grunde legte, das subcutan applicirt die unangenehmen Eigenschaften der Inunctionen mit grauer Salbe nicht besitzen und doch den nämlichen curativen Effect erzielen sollte (Wiener med. Wochenschr. 1888, Nr. 22, Wiener klin. Wochenschr. Nr. 8 und 9). Das Präparat stellt nämlich die grane Salbe selbst, nur in einer zum Zwecke der subcutanen Injection anderen Form dar. Graue Salbe, welche aus gleichen Theilen metallischen Quecksilbers und Lanolin bereitet ist, wird mit reinem Olivenöl verrieben, so dass das ganze Präparat einen Quecksilbergehalt von 30 Procent hat. Das Recept lautet:

Rp. Hydrarg.

Lanolin. āā $3 \cdot 0$

01. oliv. $4 \cdot 0$

M. D. S. zur Injection.

1 Cem. diesès grauen Oeles enthält 0.3 Qnecksilber. Der Autor empfiehlt $0.3 \mathrm{Cem}$. für die Woche zu injiciren und zwar 
an zwei Stellen des Rückens und der Nates 2-3 Wochen hindurch.

Die angewendete Menge bei den einzelnen Injectionen soll $0 \cdot 1-0 \cdot 15 \mathrm{Ccm}$. betragen. Nach seinen Angaben genügten $1.5-2 \mathrm{Ccm}$. um die Symptome zum Schwinden zu bringen. Was die Begleiterscheinungen betrifft, so fand Lang, dass die mercuriellen Schleimhauterkrankungen bei vorsichtiger Application selten auftraten und an der Einstichstelle selbst nur geringe Reactionen sich zeigten, die aber niemals in Vereiterung übergingen, weshalb er die Schlussfolgerung aussprach, dass das Oleum cinereum unter allen wirksamen Quecksilberpräparaten die geringsto Reaction hervorrufe.

Besonders günstig waren die Erfolge, welche er bei schweren Luesformen erzielte. Trost (Wiener med. Wochenschr. 1888, Nr. 18) injicirte wöchentlich 0.02 und hatte Anfangs bei vielen Kranken schmerzhafte Infiltrationen, in einigen Fällen sogar Vereiterung der Injectionsstelle zu verzeichnen, so dass er die Behandlung aussetzen musste; erst als er mit peinlichster Sorgfalt die Reinigung der Injectionsspritze selbst vollzog, fand er bei 1100 Injectionen selten derbe, empfindliche Infiltrate, nie eine Abscedirung. Er gibt an, dass zur Vermeidung der unangenehmen Reactionen das Präparat vom Apotheker mit der grössten Reinlichkeit in desinficirten Gefässen etc. bereitet und die Spritze selbst gerade bei Anwendung dieses Mittels einer besonderen Sorgfalt unterzogen werden muss, da namentlich der obere Theil des Glascylinders sehr schwer von dem anhaltenden und leicht in Zersetzung übergehenden Oele zu reinigen sei. Doutrelepont (Deutsche med. Wochenschr. 1888, Nr. 38) und Teleki (Wiener klin. Wochenschr. 1888, Nr. 8) können die günstigen Resultate mit dem Präparate bestätigen, doch bemerkten sie einige Tage lang anhaltende Infiltrate, die jedoch keine ernsteren Folgen annahmen.

Bemerkt muss noch werden, dass Lang soin Präparat auch zuir Localbehandlung empfiehlt, wo graues Pflaster ind graue Salbe nicht zur. Anwendung kommen können, so namentlich bei luetischen Processen in der Nasenhöhle, im äusseren Gehörgange, am Trommelfell, in der Tuba Eustachii, im Kehlkopf und auch bei luetischen Angenaffectionen. So heilte er einen Patienten, der in der Tibia eine grosse gummöse Höhle hatte, die mit der 
äusseren Haut nur durch eine kleine, enge Fistel in Verbindung stand. Diese locale Anwendung des grauen Oeles wurde auf der hiesigen Abtheilung nicht versucht. Auch Neisser empfiehlt (Zeitschr. X. 2. 1888)' das Ol. cin. zu Nebencuren.

Die Injectionen wurden hier tief in die Glutëen, abwechselnd rechts und links, mit besonderer Vermeidung von früheren Einstichstellen, die noch ziemlich lange durch kleine, derbe Knoten zu erkennen waren, gemacht und zwar zu $0.15 \mathrm{Ccm}$., das heisst an der Pravaz'schen Spritze wurden etwa $0.2 \mathrm{Ccm}$. eingestellt, da doch ein kleiner Theil bei der Injection verloren geht. Nach durchschnittlich 8 Injectionen bekamen die Patienten Jodkali, wie dies auch bei den anderen Mothoden geschah, und auf etwaige noch geschwollene Drüsen (meist waren es die Inguinaldrüsen, die am läugsten der allgemeinen Behandlung widerstanden) graues Pflaster. Bei sehr vielen Patienten kam es an der Einstichstelle nach zwei bis drei Tagen zu dicken, stark gorötheten Knoten, die zum Theil sehr schmerzhaft waren; die Schmerzen hörten meist schon nach einmaliger Faradisation mit secundärem Strome auf. Trotz der peinlichsten Desinfection der Spritze und der Einstichstelle wurden Abscesse beobachtet; besonder's auffallond war $\theta s$, dass einmal fast sämmtliche Patienten, die einige Tage vorher gleichzeitig Injectionen erhalten hatten, Abscesse bekamen, obgleich vorher alle Cautelen beobachtet waren und auch das graue Oel immer nur ganz frisch bereitet benutzt wurde; man konnte diese Erscheinung nur durch irgendwelche Unreinlichkeit des damals benutzten Präparates erklären. Die Wirkung liess namentlich in leichteren Fällen nichts zu wünschen übrig, in schwereren war etwas längere Zeit und 11-12 Injectionen nöthig. 25 Patienten konnten als temporär geheilt entlassen werden; davon kamen bis Ende September 18894 in 3 bis 4 Monaten wieder mit Recidiven $=16$ Procent.

Vor anderen Mitteln hat das Ol. ciner. den Vorzug, dass nur wenige Injectionen nothwendig sind, um die syphilitischen Symptome zur Involution zu bringen; doch hat es wiederum den sehr grossen Nachtheil, dass es sich nur kurze Zeit hält und mit peinlichster Sorgfalt bereitet werden muss, um Nebenerscheinungen zu vermeiden. Die Recidive lassen hier keinen sicheren Schluss zu, da ja erst 9 Monate nach der Behandlung ver- 
flossen sind, höchstens insofern, als trotz kurzer Beobachtungszeit der Procentsatz grösser ist, als bei Mitteln mit jahrelanger $\mathrm{Be}$ obachtungszeit.

Die neueste Behandlungsmethode auf der hiesigen Abtheilung besteht in intramusculären Injectionen mit Hydrarg. sozojodolicum (0.3 zu 10.0 Aq. dest. tgl. 1 Spritze); betreffs der Erfolge hiemit muss auf eine spätere Dissertation verwesen werden.

Wonn wir nun die seit 1880 hier angewendeten Syphilistherapien mit Quecksilberpräparaten nach dem Procentsatz der beobachteten Recidive ordnen, so erhalten wir folgende Reihenfolge :

Inunctionen mit Ung. hydr. cin. 9 Procent.

Quecksilberchlorid-Chlornatrium 13 Procent.

(7 gemischt mit Inunctionen 2 Recid.)

Hydrarg. salicylicum 15 Procent.

(1 gemischt mit Inunct. 1 Recid.)

Hydrarg. formamidatum 16 Procent.

(34 gemischt mit Inunct. 8 Recid.)

Hydrarg. peptonatum 16 Procent.

(42 gemischt mit Inunct. 8 Recid.)

Oleum cinerum 16 Procent.

Hydrarg. tannicum oxydulatum 18 Procent.

Hydrarg. oxydatum carbolicum 27 Procent.

Den geringsten Procentsatz weisen somit die Inunctionen auf, obwohl gerade hier die Beobachtungszeit am längsten war.

Von den vielen Quecksilberpräparaten, die im Laufe der Zeit entstanden sind und sämmtliche die Hauptaufgabe hatten, die Inunctionen mit grauer Salbe zu ersetzen, sind hier sowohl lösliche wie unlösliche Präparate, subcutane, wie innere Mittel zur Anwendung gekommen, so dass wir von den unzähligen angegebenen Mitteln so ziemlich die Hauptvertreter der einzelnen Gruppen kennen lernten.

Die Vortheile, die man mit der Einführung der Injectionen und internen Anwendung erreichte, sind keine unwesentlichen; vor Allem ist die Application eine viel einfachere und bequemere und doch wissenschaftlichere geworden, da man genau die aufgenommene Menge Quecksilber bestimmen kann, was bei den Ein- 
reibungen nicht möglich ist, wenigstens bei den leicht löslichen und schnell in den Organismus gelangenden Mitteln, während bei den schwer löslichen ein grosser Theil der Injectionsflüssigkeit lange Zeit, ohne resorbirt zu werden, liegen bleibt, wodurch natürlich ebensowenig eine Bestimmung der aufgenommenen Menge Quecksilbers nach den einzelnen Injectionen möglich ist. Alle diese nemen Methoden haben ausserdem noch den Vorzug der Reinlichkeit und der discreten Behandlung. Was jedoch den Vorwurf gegen die Inunctionen, die mercurielle Erkrankung der Mundschleimhaut betrifft, so hat man mit den neuen Präparaten keinen wesentlichen Erfolg erzielt, da fast bei jedem Mittel eine mehr oder weniger grosse Anzahl von Patienten an Stomatitis erkrankte und es ja auch bei den Inunctionen, allerdings durch sorgfältige Behandlung, möglich ist, eine Stomatitis bintanzuhalten oder wenigstens das Weitergreifen einer vorhandenen zu verhüten. Dagegen ist eine Erkrankung an Stomatitis von Leuten in der Umgebung des Patienten natürlich bei Injectionen und innerer Verabreichung nicht möglich, ebensowenig das Auftreten von Eczem, wie es auf den eingeriebenen Stellen manchmal auftritt, jedoch das eigentliche Mercurialexanthem, das ja grösstentheils überhaupt auf einer Prädisposition berulit, kann auch durch die subcutanen und inneren Mittel nicht vermieden werden. Die letzteren, die ja in Frankreich so beliebt sind, haben sich bei uns nicht eïnbürgern können, da sie die üblen Nebenerscheinungen von Seiten des Intestinaltractus zur Folge haben, die selbst bei den leichtverträglichsten MitteIn, wie bei Verabreichung des Hydrarg. oxydul. tannic, beobachtet wurden. Da hiebei ein grosser Theil des Quecksilbers nicht zur Rosorption gelangt, so ist man über die Aufnahme der Quecksilbermenge ebenso im Unklaren, wie bei den Inunctionen, und so kann die Bohandlung mit inneren Mitteln nie als gleichwerthiger Ersatz der Einreibungen gelten.

Ebenso haben die Injectionsmethoden ibre Vortheile und Nachtheile. Da das Quecksilber bei dieser Application nicht von einer grossen Fläche der äusseren Haut, sondern von einer einzigen Stelle ans in den Organismus gelangt, so muss diese örtliche Anhäufung, da ja anch das Mittel concentrirt genug sein muss, um die vielen Quecksilbertheilchen zu ersetzen, die bei der Einreibung resorbirt werden, zu einer entzündlichen Reaction führen; 
und gerade diejenigen Mittel, bei denen wegen ihrer chemischen Zusammensetzung die moiste Garantie geboten ist, dass keine Reaction an der Einstichstelle eintritt, bleiben umsomehr hinter der Wirknng der Inunctionen zurück, während die an Wirkung den Einreibungen am nächsten stehenden Mittel, wie Calomel sehr bäufige Reactionen und Abscesse zur Folge haben. Diese Reactionsknoten sind zum Theile sehr schmerzhaft, und immer ist die Gefahr gegeben, dass sie sich in Abscesse umwandeln, sei es nun, dass die Elemente des lebenden, organischen Gewebes, wie Mesterton (Schmitt's Jahrb. 1880) mejnt, durch die Entziehung des zur Verbindung mit dem Quecksilber nothwendigen Eiweiss nekrotisiren, oder dass durch Infection von aussen, durch Anwendung eines unreinen Präparates Abscesse entstehen. Ein grosser Fortschritt wurde deshalb durch die Anwendung intramusculärer Injectionen nach dem Yorgange ron Smirnhoff gemacht, indem der ins Muskelfleisch eingeschlossene Injectionsherd äusseren Schädlichkeiten weniger zugänglich ist, die Schmerzen wegen der geringen Empfindlichkeit der Muskeln geringer sind, und wegen der Dicke der Haut und Muskeldecke eine Ulceration nicht gut eintreten kann. Und doch sind auch bei diesen Injectionen schon sehr schmerzhafte Entzündungen und Abscesse beobachtet worden.

Trotzdem, die unlöslichen Präparate zum grossen Theil entzündliche Reactionen an der Injectionsstelle und Abscedirungen im Gefolge haben, sind ihre Erfolge noch besser, als die, welche man mit den löslichen erzielte, die schnell in den Kreislauf gelangen, den Organismus förmlich überfluthen, aber ebenso rasch wieder aus dem Körper verschwinden, da ihnen meist durch ihre chemische Zusammensetzung die Kraft fehlt, sich im Gowebe an Eiweisskörper zu binden und so durch längeres Verweilen im Körper eine längere Einwirkung auf dēn allgemeinen Krankheitsprocess auszuüben. Sie eignen sich in Folge dessen nur, da aber vorzüglich, für die Anwendung bei leichteren Formen von Syphilis.

Heutzutage besteht kein Zweifel mehr, dass das Auftreten von Recidiven am ehesten durch Curen verhütet werden kann, welche die Eigenschaft besitzen, den Körper möglichst lang unter dem Eỉnflusse des Quecksilbers zu halten. Während nach Inunc- 
742 Lex r. Der Werth der verschied. Quecksilberpräyarate in der Syphilistherapie.

tionscuren woch nach mindestens 6 Monaten Quecksilber im Harne nachzuweisen ist, treffen auf Pepton 10-11 Wochen, auf Formamid nur 6 Wochen. (Bockhardt, Monatsschr. f. prakt. Derm., Nr. 5, 1885.) Ferner ist noch zu erwähnen, dass bei den meisten hier angewendeten Injectionscuren die Behandlung mit Quecksilberpflaster nothwendig war, um die Adenitiden vollständig zum Zurückgehen zu bringen.

Während beim Emporkommen der Injectionsmethoden die Inunctionen viele Anhänger verloren haben, steht hente die Mehrzahl der Forscher und Aerzte wieder auf ihrer Seite, indem man sie zwar für eine roh empirische, unwissenschaftliche Methode, aber immer noch für die wirksamste Cur hält. Dagegen hat das Streben, die grane Salbe zu verdrängen und durch bessere Präparate zu ersetzen, eine Reihe von Quecksilberverbindungen hervorgebracht, die zwar alle ihre Vortheile vor den Inunctionen, aber auch wieder ihre eigenthümlichen Nachtheile besitzen, abgesehen davon, dass sie in ihrer Wirkung die Inunctionen nicht erreicht oder übertroffen haben.

Wohl sind unter den Injectionspräparaten manche, wie Calomel oder Sublimat, die in Fällen, wo sich eine Inunctionscur nicht gut und sicher durchführen lässt, ganz gute Dienste leisten, auch solche, und das ist die Mehrzahl, die in leichteren Fällen mit gutem Erfolg angewendet werden können, bei schwereren jedoch zu schwach sind; allein, so lange es nicht gelungen ist, für irgend eine Applicationsmethode ein Präparat zu finden, das sämmtlichen Auforderungen gerecht wird, ohne wieder neue Nachtheile aufzuweisen, worden die Inunctionen mit grauer Salbe immer noch die sicherste Gewähr für Heilung und möglichst grosse Verminderung der Recidive bieten.

Zum Schlusse erlaube ich mir, für die Anregung zu dieser Arbeit, sowie für die Unterstützung bei Ausführung derselben den Herren Privatdocent Dr. Seifert und Dr. du Mesnil meinen besten Dank auszusprechen. 